\title{
Prevention of multidrug-resistant infections from contaminated duodenoscopes: Position Statement of the European Society of Gastrointestinal Endoscopy (ESGE) and European Society of Gastroenterology Nurses and Associates (ESGENA)
}

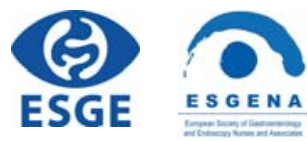

Authors

Ulrike Beilenhoff ${ }^{1}$, Holger Biering ${ }^{2}$, Reinhard Blum ${ }^{3}$, Jadranka Brljak ${ }^{4}$, Monica Cimbro ${ }^{5}$, Jean-Marc Dumonceau ${ }^{6}$, Cesare Hassan ${ }^{7}$, Michael Jung $^{8}$, Christiane Neumann ${ }^{9}$, Michael Pietsch ${ }^{10}$, Lionel Pineau ${ }^{11}$, Thierry Ponchon ${ }^{12}$, Stanislav Rejchrt ${ }^{13}$, Jean-François Rey ${ }^{14}$, Verona Schmidt ${ }^{15}$, Jayne Tillett ${ }^{16}$, Jeanin van Hooft ${ }^{17}$

Institutions

1 ESGENA Scientific Secretary, Ulm, Germany

2 Grevenbroich, Germany

3 Olympus Europa, Hamburg, Germany

4 University Hospital KBC-Zagreb-Rebro, Zagreb, Croatia

5 Medical Devices Division, CBC Europe Srl, Nova Milanese (MB), Italy

6 Gedyt Endoscopy Center, Buenos Aires, Argentina

7 Digestive Endoscopy Unit, Catholic University, Rome, Italy

8 2nd Department of Internal Medicine, Katholisches Klinikum, Mainz, Germany

9 ESGENA Past President, Birmingham, UK

10 Department of Hygiene and Infection Prevention, Medical Center, University Hospital, Mainz, Germany

11 Biotech Germande, Marseille, France

12 Digestive Diseases Department, Hôpital Edouard Herriot, Lyon, France

13 2nd Department of Internal Medicine, Charles University Teaching Hospital, Hradec Králové, Czech Republic

14 Institut Arnault Tzanck, St. Laurent du Var, France

15 Microbiology and Hygiene Department, Chemische Fabrik Dr. Weigert, Hamburg, Germany

16 St. Woolos Hospital, Newport, UK

17 Department of Gastroenterology and Hepatology, Academic Medical Center, Amsterdam, The Netherlands

Bibliography

DOI https://doi.org/10.1055/s-0043-120523 | Published online: 16.10.2017 | Endoscopy 2017; 49: 1098-1106

(c) Georg Thieme Verlag KG Stuttgart · New York

ISSN 0013-726X
Corresponding author

Ulrike Beilenhoff, ESGENA Scientific Secretariat, Ferdinand-Sauerbruch-Weg 16, 89075 Ulm, Germany info@esgena.org

\section{RECOMMENDATIONS}

Patients should be informed about the benefits and risks of endoscopic retrograde cholangiopancreatography (ERCP)

Only specially trained and competent personnel should carry out endoscope reprocessing.

Manufacturers of duodenoscopes should provide detailed instructions on how to use and reprocess their equipment.

In the case of modifications to their equipment, manufacturers should provide updated instructions for use.

Detailed reprocessing protocols based on the manufacturer's instructions for use should clearly lay out the different reprocessing steps necessary for each endoscope model.

Appropriate cleaning equipment should be used for duodenoscopes in compliance with the manufacturer's instructions for use.

Only purpose-designed, endoscope type-specific, singleuse cleaning brushes should be used, to ensure optimal cleaning.

As soon as the endoscope is withdrawn from the patient, bedside cleaning should be performed, followed by leak testing, thorough manual cleaning steps, and automated reprocessing, in order to: 
- Remove debris from external and internal surfaces;

- Prevent any drying of body fluids, blood, or debris;

- Prevent any formation of biofilms.

In addition to the leak test, visual inspection of the distal end as well as regular maintenance of duodenoscopes should be performed according to the manufacturer's instructions for use, in order to detect any damage at an early stage.

The entire reprocessing procedure in endoscope washerdisinfectors (EWDs) should be validated according to the European and International Standard, EN ISO 15883.

Routine technical tests of EWDs should be performed according to the validation reports.

\section{GLOSSARY}

Adenosine triphosphate (ATP) A substance present in all living cells that provides energy for many metabolic processes and is involved in making ribonucleic acid.

Clinical care provider An organization, person or group of persons legally responsible for the provision of a clinical service. This could be an institution, hospital or department, or a doctor working on his/her own premises. Performance qualification Final inspections and tests to confirm that an endoscope washer-disinfector is effective for endoscope reprocessing in the environment at the reprocessing area. It is based on the European and International Standard EN ISO 15883.
ABBREVIATIONS
ERCP endoscopic retrograde cholangio- pancreatography
EWD endoscope washer-disinfector
FDA [US] Food and Drug Administration
GI gastrointestinal
MDRO multidrug-resistant organism
SOP standard operating procedure

This Position Statement of the European Society of Gastrointestinal Endoscopy (ESGE) and European Society of Gastroenterology Nurses and Associates (ESGENA) addresses the recent outbreaks of infection with multidrug-resistant organisms following endoscopic retrograde cholangiopancreatography, and proposes measures to prevent or manage such outbreaks. As a consequence of the outbreaks,
Microbiological surveillance of a proportion of the department's endoscopes should be performed every 3 months, with the requirement that all endoscopes used in the unit are tested at least once a year.

In the case of suspected endoscopy-related infection, the relevant device (e.g., endoscope, EWD) should be taken out of service until adequate corrective actions have been taken.

Outbreaks should be managed by a multidisciplinary team, including endoscopy, hygiene, and microbiology experts, manufacturers, and regulatory bodies, according to national standards and/or laws.

In the case of suspected multidrug-resistant organism (MDRO) outbreaks, close cooperation between the endoscopy unit and the clinical health provider is essential (including infection control departments and hospital hygienists). the development of purpose-designed cleaning equipment has been intensified, and manufacturers have provided more instructions on how to reprocess duodenoscopes appropriately and how to identify when servicing is necessary. The Position Statement addresses the importance of staff training, reprocessing and maintenance of duodenoscopes, process validation, and microbiological surveillance, as well as outbreak management.

\section{Introduction}

Since its introduction more than 40 years ago, endoscopic retrograde cholangiopancreatography (ERCP) has fundamentally changed the treatment of biliopancreatic diseases. The procedure is considered standard for the treatment of common bile duct stones and of tumors obstructing the biliary tract, and may be applied for pancreatic duct stones or strictures. Since the late 1970 s there have been sporadic reports of nosocomial infections linked to endoscopic procedures, the majority caused by inadequate reprocessing of endoscopes [1].

Infections due to multidrug-resistant organisms (MDROs) however have increasingly become a concern in health care, including in gastrointestinal (GI) endoscopy [2]. Since 2010, reports of individual cases and serial outbreaks of MDRO infections associated with ERCP have been published in the United States of America (USA), France, Germany and the Netherlands [3-8]. In addition, there have been cases and series of outbreaks that have only been reported to national regulatory bodies in the respective countries [2,9-12].

Screening and identifying patients who are carriers of infective organisms is often problematic. MDROs can be transmitted from one patient to another, or, as the human gut can be regarded as a bacterial reservoir, within patients themselves. The hospital environment (hands, doorhandles, furniture, toilets, bathroom facilities, etc) also poses a potential infection risk if 
not cleaned/disinfected appropriately. A key component in the chain of infection is often poor hand hygiene of clinical staff, visitors, and/or patients.

Because of limited antibiotic therapeutic options, infections with MDROs may result in poor clinical outcomes. Carbapenem-resistant enterobacteria are MDROs that produce carbapenemases - enzymes that hydrolyze the beta-lactam ring and inactivate beta-lactam antibiotics [2]. Several strains of carbapenemase-producing intestinal bacteria have been detected after outbreaks related to ERCP [13]. Immunocompromised patients, especially those with cholangiocarcinoma and biliary stents, are at increased risk of acquiring these infections, often with resulting poor outcomes $[2,13,14]$. Despite the risk, these immunocompromised patients potentially may benefit significantly from therapeutic ERCP.

As a result of the MDRO outbreaks associated with ERCP, the European Society of Gastrointestinal Endoscopy (ESGE) and the European Society of Gastroenterology Nurses and Associates (ESGENA) formulated this Position Statement to give advice regarding the reprocessing of duodenoscopes.

\section{Method}

This ESGE-ESGENA Position Statement is based on a multidisciplinary consensus from an expert working group consisting of gastroenterologists, endoscopy nurses, chemists, microbiologists, and industry representatives, with experience in developing national and international guidelines in hygiene and infection control.

A literature search was carried out evaluating published reports of ERCP-related outbreaks, reviews, and national guidelines, during the period 2010-2016.

Based on the assessment of the literature reviews and advice from various official national bodies, this Position Statement reflects expert opinion on what constitutes good clinical practice $[15,16]$. The quality of evidence and strength of recommendations were not formally graded [17] as they were generally low.

The authors met twice during 2016. A consensus document was agreed in 2017. The manuscript was sent to all ESGE and ESGENA member societies and their individual members and to two ESGE Governing Board members for approval, resulting in this final version, agreed by all authors.

\section{Literature review and questions raised by the outbreaks}

Although most of the duodenoscopes involved were made by Olympus, it is important to emphasize that duodenoscopes from all major manufacturers (Olympus, Pentax, Fujifilm) were involved in the reported outbreaks.

The reported outbreaks and the publications featured the following issues:

- Multidrug-resistant Enterobacteriaceae (including Klebsiella pneumoniae and Escherichia coli) and Pseudomonas aeruginosa were found in duodenoscopes, especially at the distal end, around the forceps elevator mechanism [2-13].
- One outbreak was promoted by insufficient cleaning and drying of endoscopes [3], while other outbreaks occurred in spite of apparently appropriate reprocessing protocols $[2,4-8,13]$. This raises a question about the efficacy of the reprocessing procedures for flexible endoscopes.

$\mathrm{Gl}$ endoscopes, including duodenoscopes, may have a bacterial burden of $10^{8-10}$, or $8-10 \log _{10}$ on a logarithmic scale, before reprocessing. Manual cleaning plus machine reprocessing leads to an $8-12 \log _{10}$ reduction of the microbial load. Consequently, the safety margin is narrow at $0-2$ $\log _{10}[18]$, and not achieved if the reprocessing is not carried out in line with the decontamination protocols. Therefore, even minor deviations from reprocessing protocols can result in potentially infective materials remaining in parts of the endoscope, which consequently increases the risk of cross-contamination.

- In several outbreak series, epidemiological investigations identified the duodenoscopes as the potential source of the outbreak, but the outbreak strains could not be cultivated from all the suspected endoscopes. This may support the hypothesis that periodic rather than continual irregularities in reprocessing caused these outbreaks $[2,7,11-13,18]$. Endoscope reprocessing includes a number of manual cleaning steps. Human error and inaccurate handling can leave residual debris on and in the instruments after manual cleaning, which increases the risk of inadequate decontamination.

- In many outbreaks, small cracks at the distal end of the duodenoscopes were identified when the suspected endoscopes were sent for maintenance or repair [4-8]. Small defects were identified behind glass lenses and around the elevator mechanisms, which could have been the entry points for bacteria and subsequent biofilm formation. This raises the question of whether the leak test can accurately identify these micro defects or whether the leak tests were not carried out according to manufacturers' recommendations soon after the procedures.

- Because of the lateral position of the lens, duodenoscopes are more likely to be exposed to mechanical stress than gastroscopes or colonoscopes, which may be one reason for the occurrence of the aforementioned defects. The elevator mechanism and the narrow lumen behind the fixed distal caps of duodenoscopes are difficult to access with standard cleaning brushes $[3,4,8]$.

- In many reports the quality and appropriateness of the cleaning brushes used and their ability to access all parts of the distal tips of the duodenoscopes were identified as crucial in preventing cross-contamination [2-8]. The cleaning brush size needs to be appropriate for the size of the instrument/suction channel and other components. The brushes should have adequate bristles to ensure thorough cleaning in line with manufacturers' recommendations.

- Insufficient hand hygiene and cleaning/disinfection of the patient environment were also identified as factors favoring bacterial patient-to-patient transmission [7]. Carbapenemresistant Enterobacteriaceae infections were transmitted 
from one patient to another whether they had undergone ERCP or not.

- Reviews and editorials discussed the outbreaks and their consequences without agreeing on a single identifiable cause but rather pointing to multiple factors $[2,11-13$, $18,19]$.

- In one hospital, despite apparently appropriate reprocessing, $2 \%$ of duodenoscopes were found to be contaminated with nonpathogenic and pathogenic germs [20]. As an additional step, all reprocessed duodenoscopes were then quarantined for 48 hours before the next use, until negative microbiological cultures were obtained [20].

In the reported cases the outbreaks were stopped by the following measures:

- The reprocessing procedure was reviewed and improved [3].

- The suspected duodenoscopes were taken out of service and necessary investigations were initiated [3-8].

- Contaminated duodenoscopes were sent for maintenance, repair, and replacement of single components, even though micro defects had not been identified by endoscopy staff during leak testing $[4,7,8]$.

- At one center, duodenoscopes were sterilized with ethylene oxide after conventional cleaning and disinfection. This did not lead to a satisfactory reduction in micro-organisms [4].

\section{Reactions from regulatory bodies}

In the USA the reported outbreaks drew considerable media attention. The Food and Drug Administration (FDA) published several statements to raise awareness among health care professionals that the complex design of duodenoscopes might impede effective reprocessing [9]. Official bodies and professional societies in various European countries (e.g., France, Germany, The Netherlands) also informed their health care providers accordingly, initiated appropriate actions, and issued recommendations on how to:

- Prevent cross-contamination;

- Improve/ensure effective reprocessing;

- Manage outbreaks.

\section{Recommendations}

In response to the reported outbreaks, ESGE and ESGENA make the following recommendations.

\section{Patient information and informed consent}

\section{RECOMMENDATION}

Patients should be informed about the benefits and risks of ERCP.

It is undisputed that many biliopancreatic diseases, including life-threatening conditions, can be successfully managed by ERCP. The risk of ERCP-related infections is relatively low compared to the potential benefits. Consequently, national regulatory bodies in the USA and in Europe recommend not to discontinue carrying out ERCPs, but to inform patients about the potential risk of infections during these procedures $[9,10]$.

\section{Training, awareness, and professional duty}

\section{RECOMMENDATION}

Only specially trained and competent personnel should carry out endoscope reprocessing.

\section{RECOMMENDATION}

Manufacturers of duodenoscopes should provide detailed instructions on how to use and reprocess their equipment.

\section{RECOMMENDATION}

In the case of modifications to their equipment, manufacturers should provide updated instructions for use.

Reprocessing of medical devices requires specialized knowledge and skills, as emphasized by European and national guidelines and national laws/standards. It is the responsibility of the clinical service provider to ensure the training and competence of staff involved in the reprocessing of equipment [21-26], and to provide adequate staffing levels in addition to space and reprocessing equipment (structure quality) to enable reprocessing according to manufacturers' instructions. Staff should be aware of their responsibilities in reprocessing complex medical devices.

Reprocessing staff need to be familiar with:

- The specific design of each endoscope model used in a department, including endoscopes on loan;

- Infection prevention policies, including health and safety issues and hand hygiene;

- Endoscope decontamination processes, including all essential steps of cleaning, disinfection, drying, storage, documentation, and traceability.

Officially recognized training courses and systems of ongoing competency assessment are available in many European countries. ESGENA has developed a European Curriculum that includes the training and competencies required in reprocessing of endoscopes [26].

Reviews of outbreaks showed that the potential risk of infections increases if national guidelines and local protocols are not strictly followed [1,2]. Therefore, periodic audits of the reprocessing procedures as well as periodic training sessions and competency assessments are helpful tools to identify deficiencies, to implement improvements, and to ensure up-to-date practices. 
If new endoscopes are introduced into an endoscopy unit, it is the manufacturer's legal responsibility to provide updated information on appropriate reprocessing protocols [27].

If health care professionals (physicians, nurses, reprocessing experts) have the impression that new or changed designs hinder or prevent safe reprocessing, it is their professional responsibility to inform the service provider and/or the manufacturer about these difficulties. If the problem persists, regulatory bodies must be informed.

\section{Reprocessing and maintenance of duodenoscopes}

\section{RECOMMENDATION}

Detailed reprocessing protocols based on the manufacturer's instructions for use should clearly lay out the different reprocessing steps necessary for each endoscope model.

\section{RECOMMENDATION}

Appropriate cleaning equipment should be used for duodenoscopes in compliance with the manufacturer's instructions for use.

\section{RECOMMENDATION}

Only purpose-designed, endoscope type-specific, singleuse cleaning brushes should be used, to ensure optimal cleaning.

\section{RECOMMENDATION}

As soon as the endoscope is withdrawn from the patient, bedside cleaning should be performed, followed by leak testing, thorough manual cleaning steps, and automated reprocessing, in order to

- Remove debris from external and internal surfaces;

- Prevent any drying of body fluids, blood, or debris;

- Prevent any formation of biofilms.

\section{RECOMMENDATION}

In addition to the leak test, visual inspection of the distal end as well as regular maintenance of duodenoscopes should be performed according to the manufacturer's instructions for use, in order to detect any damage at an early stage.

Even if automated reprocessing is performed, a thorough bedside cleaning followed by full manual cleaning according to the manufacturer's instructions for use is essential, paying special attention to appropriate brushing and detergent flushing of all channels.
Thorough cleaning is a prerequisite for effective disinfection. Any deviation from guidelines and protocols may result in failure of the reprocessing procedure, as infection outbreaks have demonstrated [1,2]. Bedside cleaning and the manual cleaning steps with flushing and brushing of the entire channel systems are the most important steps for the removal of debris, blood, and body fluids. Remaining protein debris can become fixed by drying or by using inappropriate chemicals. Biofilm formation is possible if the cleaning and rinsing steps have not been carried out correctly. As some Gram-negative bacteria can undergo cell division every 20 to 30 minutes, it is essential to complete all reprocessing steps quickly, before bacterial growth and debris begin to dry on surfaces thereby promoting the formation of biofilms $[25,28]$. Micro-organisms embedded in biofilms are 10 to 100 times more resistant to process chemicals than planktonic (free-floating) micro-organisms [29], and are frequently released from biofilms. Some national guidelines recommend performance of all manual reprocessing steps within 30 minutes after completion of the patient examination $[25,30]$.

In response to the recent outbreaks, endoscope manufacturers have reviewed and updated their reprocessing instructions for duodenoscopes and have consequently alerted their customers [9, 23].

Cleaning brushes are themselves difficult to clean and they potentially carry over organic material, and damaged brushes may damage the equipment channels. Therefore it is strongly recommended to use new single-use cleaning brushes for each reprocessing cycle in order to prevent cross-contamination and damage to the endoscopes [21]. All guidelines emphasize the need for thorough brushing of all accessible channels [21-25, 31]. Studies have shown the positive effect of multiple cleaning/brushing procedures [32]. However it is difficult to calculate the exact optimal number of brushing cycles, as the germ load varies greatly from patient to patient.

The currently available duodenoscopes differ in the design of their distal tip and their elevator mechanism, depending on the manufacturer and the endoscope generation (see $\triangleright$ Table 1). The differences between duodenoscopes, according to model and manufacturer, must be taken into account during reprocessing, and the different types require different approaches for cleaning. The following general recommendations may serve as a guide.

\section{- Duodenoscopes with accessible elevator wire channels.}

If the elevator wire channel is accessible, it is essential to flush the channel immediately by using dedicated cleaning adapters upon withdrawal. It is necessary to connect it correctly in the endoscope washer-disinfector (EWD) as well as in the drying cabinets (if available). In addition the elevator lever should be positioned according to EWD manufacturer instructions (e.g. at a $45^{\circ}$ angle).

- Duodenoscopes with removable distal caps. Removable distal caps and distal ends make cleaning easier as they enable free access for brushes to the elevator mechanism. Reusable detachable caps also need a thorough manual cleaning before automated reprocessing.

The caps remain detached during automated reprocessing, 
drying, and storage but the removable parts need careful reattachment before use.

If detachable components of the duodenoscope can be autoclaved, thorough manual cleaning prior to autoclaving is also a prerequisite for successful sterilization.

- Duodenoscopes with fixed distal caps. Fixed distal ends demand more attention during cleaning because the narrow lumen behind the elevator is difficult to access with standard cleaning brushes [2-7]. Special brushes are needed in order to ensure thorough cleaning and to avoid any damage. Various manufacturers have launched specially designed cleaning brushes for duodenoscopes. Manufacturers (either of endoscopes or of cleaning accessories) should provide relevant data about the compatibility of their cleaning devices [2].

Table 1 Different designs of the distal tips of duodenoscopes.

- Fixed distal end and a rinsable elevator wire channel

- Fixed distal end and a sealed elevator wire channel

- Removable distal cap and rinsable elevator wire channel

- Removable distal end that can be dismantled and autoclaved having a brushable elevator wire channel

In the recent ERCP-related outbreaks, small cracks, minor defects, and remaining debris with microbiological loads were detected at the distal end of some duodenoscopes during service [4-8].

Audits following the outbreaks did not discover any lapses in reprocessing procedures, but the publications did not mention whether a leak test was routinely performed manually and in the EWD [4-8].

Case reports from gastroenterology, pneumatology, and cardiology settings showed that damaged endoscopes caused a series of infections after transesophageal endoscopy and after bronchoscopy $[1,33,34]$, which underlines the importance of the leak test. This raises the question of whether the leak test can detect micro defects or not. Therefore visual inspection (using magnifying glasses) especially of the distal end of the duodenoscope for potential small defects and micro damage is recommended after completion of the entire reprocessing cycle [22]. Manufacturers already recommend periodic maintenance and routine replacement of certain components [9] to prevent harm resulting from micro damage.

Thorough drying of all endoscope channels is necessary before storage, to prevent any growth of micro-organisms such as waterborne bacteria that thrive in damp conditions.

In some countries drying cabinets are already recommended (e.g., United Kingdom, France, The Netherlands). Other countries emphasize the quality of drying (e.g., Germany).

\section{Process validation and microbiological surveillance}

\section{RECOMMENDATION}

The entire reprocessing procedure in endoscope washerdisinfectors (EWDs) should be validated according to the European and International Standard, EN ISO 15883.

\section{RECOMMENDATION}

Routine technical tests of EWDs should be performed according to the validation reports.

\section{RECOMMENDATION}

Microbiological surveillance of a proportion of each department's endoscopes should be performed every 3 months, with the requirement that all endoscopes used in the unit are tested at least once a year.

The validation of the entire reprocessing procedure is a prerequisite for an effective and reproducible process $[35,36]$. It is important that the Performance qualification (see Glossary) is done with all the different endoscope types used in each department, in order to prove the efficacy of the reprocessing cycle with all endoscopes [35, 36].

Routine microbiological surveillance is important to identify any deviations, technical errors, and failures of the reprocessing cycle [35-37]. The ESGE-ESGENA Guideline recommends regular microbiological surveillance every 3 months and emphasizes the necessity of sampling all parts and available channels of an endoscope [37].

After the recent outbreaks, the US Centers for Disease Control have published interim recommendations on microbiological surveillance for duodenoscopes. However, these do not cover all parts and channels of duodenoscopes [38].

The frequencies and test methods for microbiological surveillance are contentious and are the focus of controversial discussions in various publications; these issues need further research $[2,11,12,23]$.

In the USA, additional tests for detecting residual tissue markers (e.g., ATP, hemoglobin) have been introduced, with the aim of validating the efficacy of manual cleaning before disinfection $[2,22,23]$. 
- Table2 Quality criteria for the safe reprocessing of endoscopes.

\section{Structure quality}

\begin{tabular}{|l|l|l|}
\hline Reprocessing room & $\begin{array}{l}\text { - Separate, purpose-designed, reprocessing room } \\
\text { - Strict spatial and procedural separation between contaminated and clean working areas and workflow, with } \\
\text { a preference for two separate rooms }\end{array}$ \\
\hline Staff number & - Adequate number of competent staff \\
\hline Staff qualifications & - Only trained and competent staff perform endoscope reprocessing \\
& - Structured training program, regular competency assessment, and updates \\
& - Documentation of initial training, updates, and regular competency assessments
\end{tabular}

\section{Process quality}

Standard operating procedure (SOP) for reprocessing

- Detailed reprocessing protocols according to manufacturer's recommendations, including photos, for each different type of endoscope available in the department, with easily accessible copies also in the reprocessing room

- Regular updates of SOPs, adopting new evidence and product changes

- Archiving of documented reprocessing cycle for each patient

Precleaning in endoscopy room

- Rinsing/flushing of all accessible channels with detergents by using dedicated cleaning adapters

- Function checks of all channels

- Transport in closed containers

Manual cleaning of endoscopes

- Manual leak testing, after each endoscopy procedure, after precleaning, and before starting the steps of the cleaning procedures

- Manual cleaning of all accessible channels and vital components using suitable brushes

- Single-use brushes only (size according to the channel diameter, approved for use with the specific gastrointestinal endoscope, new brush for each endoscope decontamination cycle)

- Special brushes for certain components (e. g., distal tip and elevator mechanism)

- Thorough cleaning of distal tip of duodenoscopes, including detachable components

- Flushing of all accessible channels with detergent, followed by rinsing with water, using dedicated adapters

- Visual assessment of cleanliness

Reprocessing in endoscope washer-disinfector (EWD)

- Correct positioning of endoscope in EWD including correct positioning of the elevator mechanism

- Correct connection of all channels

- Checking at the end of disinfection to ensure that all connections are attached and have not come loose during the cycle

- Visual assessment of cleanliness

Drying and storage

- Complete drying of all external and internal surfaces with medical compressed air

- Storage in closed, ventilated cabinets or drying/storage cabinets

Documentation

- Documentation of all manual and automated reprocessing steps, including technical parameters of the reprocessing cycle, reprocessing staff, and patient in whom the endoscope was used

- Archiving of reprocessing details (according to national laws/standards)

Release of endoscope

- Verification of correct reprocessing by checking all process parameters

- Visual inspection of distal end components, using magnifying glass, for possible defects and damage

- Documentation of release for use

\begin{tabular}{|l|l|}
\hline \multicolumn{1}{|l|}{ Outcome quality } \\
\hline $\begin{array}{l}\text { Validation of reprocessing } \\
\text { procedure }\end{array}$ & - Validation according to EN ISO 15883 once a year \\
& - Poctocols for routine technical tests \\
\hline Microbiological surveillance & - Protocol for microbiological surveillance \\
\hline Maintenance & - Documentation of microbiological surveillance \\
\hline Outbreak management & - Protocol for maintenance of all endoscopes, EWDs, and drying cabinets \\
\hline
\end{tabular}




\section{Outbreak management}

\section{RECOMMENDATION}

In the case of suspected endoscopy-related infection, the relevant device (e.g., endoscope, EWD) should be taken out of service until adequate corrective actions have been taken.

\section{RECOMMENDATION}

Outbreaks should be managed by a multidisciplinary team, including endoscopy, hygiene, and microbiology experts, manufacturers, and regulatory bodies, according to national standards and/or laws.

\section{RECOMMENDATION}

In the case of suspected MDRO outbreaks, close cooperation between the endoscopy unit and the clinical health provider is essential (including infection control departments and hospital hygienists).

If any contamination is found, or if a particular medical device is likely to be the cause of infection, it is the responsibility of the clinical service provider to take the suspected equipment out of service (e.g., endoscopes, EWDs, accessories). Corrective actions should be taken such as maintenance, review and improvement of the reprocessing cycle, and staff training. The outcome quality of the reprocessing cycle must be satisfactory before the suspected device can be used again [21,37].

The quality criteria for structure, process, and outcome quality of endoscope reprocessing are listed in $>$ Table 2 .

\section{Summary}

Safe and effective endoscope reprocessing is crucial to patient safety in endoscopy. Noncompliance with guidelines as well as deviations from standardized and validated reprocessing protocols may lead to ineffective reprocessing, with the possibility of patient-to-patient transmission. Recent MDRO outbreaks show how narrow the margin of safety is, despite compliance with reprocessing protocols. Important tools in the prevention of infections are:

- Staff training and regular competence assessment;

- Staff adherence to guidelines and to manufacturers' specifications and instructions for use;

- Routine inspections of endoscopes, and regular/scheduled maintenance;

- Periodic quality assessment, with audits of staff compliance, microbiological surveillance, and validation of reprocessing cycles;

- Dedicated places and equipment for all reprocessing steps (structure quality).
When there is suspicion of endoscopy-based transmission, infections should be managed by a multidisciplinary team. Health care professionals and manufacturers should be aware of their legal responsibility to patients to ensure their safety.

\section{Disclaimer}

ESGE/ESGENA position statements and guidelines represent a consensus of best practice based on the available evidence at the time of preparation. They may not apply in all situations and should be interpreted in the light of specific clinical situations and resource availability. Further controlled clinical studies may be needed to clarify aspects of the statements, and revision may be necessary as new data appear. Clinical consideration may justify a course of action at variance to these recommendations. ESGE/ESGENA position statements and guidelines are intended to be an educational device to provide information that may assist endoscopists in providing care to patients. They are not rules and should not be construed as establishing a legal standard of care or as encouraging, advocating, requiring, or discouraging any particular treatment.

\section{Competing interests}

U. Beilenhoff has provided consultancy to Boston Scientific since July 2017. H. Biering has done customer presentations and trainings, moderated workshops, and provided consultancy to Richard Wolf $\mathrm{GmbH}$, Olympus Europa, Dr. Weigert, and Ecolab. Reinhard Blum is employed by Olympus Europa, who hold patents on endoscopes in general; he is a member of the DIN/CEN/ISO TC/198/WG13 standards group for safe reprocessing of endoscopes. M. Cimbro has been employed by CBC Europe since 2002; she has been a consultant since 2011 to ANOTE-ANIGEA (Italian Association of Gastroenterology and Endoscopy Nurses). M. Jung's hospital has received sponsorship from Olympus Optical and other companies for an EUS/endoscopy course (22nd September 2017). M. Pietsch's department has received thirdparty funding from Olympus for a research project, from 2015 to 2017. L. Pineau has provided training on hygiene and medical device evaluation for Pentax, Olympus, and Fujifilm, from 1999 to 2017. T. Ponchon has served on an Advisory board for Fujifilm (January 2015 to October 2017). J. Brljak, J.-M. Dumonceau, C. Hassan, C. Neumann, S. Rejchrt, J.-F. Rey, V. Schmidt, J. Tillett, and J. van Hooft have no competing interests.

\section{References}

[1] Kovaleva J, Peters FTM, van der Mei HC et al. Transmission of infection by flexible gastrointestinal endoscopy and bronchoscopy. Clin Microbiol Rev 2013; 26: 231 - 254

[2] Muscarella LF. Risk of transmission of carbapenem-resistant Enterobacteriaceae and related "superbugs" during gastrointestinal endoscopy. World J Gastrointest Endosc 2014; 6: 457 - 474

[3] Aumeran C, Poincloux L, Souweine B et al. Multidrug-resistant Klebsiella pneumoniae outbreak after endoscopic retrograde cholangiopancreatography. Endoscopy 2010; 42: 895 - 899

[4] Epstein L, Hunter JC, Arwady MA et al. New Delhi metallo- $\beta$-lactamase-producing carbapenem-resistant Escherichia coli associated with exposure to duodenoscopes. JAMA 2014; 312: 1447-1455 
[5] Wendorf K, Kay M, Baliga C et al. Endoscopic retrograde cholangiopancreatography-associated AmpC Escherichia coli outbreak. Infect Control Hosp Epidemiol 2015; 36: 634-642

[6] Smith ZL, Yuong SO, Saeian K et al. Transmission of carbapenem-resistant Enterobacteriaceae during ERCP: time to revisit the current reprocessing guidelines. Gastrointest Endosc 2015; 81: 1041 - 1045

[7] Kola A, Piening B, Pape UF et al. An outbreak of carbapenem-resistant OXA-48-producing Klebsiella pneumonia associated to duodenoscopy. Antimicrob Resist Infect Control 2015; 4: 8 [doi:10.1186/s13756015-0049-4]

[8] Verfaillie C], Bruno MJ, Voor in 't holt AF et al. Withdrawal of a noveldesign duodenoscope ends outbreak of a VIM-2-producing Pseudomonas aeruginosa. Endoscopy 2015; 47: $493-502$

[9] US Food and Drug Administration (FDA). Safety Communication. Available from: http://www.fda.gov/MedicalDevices/Safety/AlertsandNotices/default.htm [Accessed: April 20 2017]

[10] Bundesinstitut für Arzneimittel und Medizinprodukte. Empfehlungen des BfArM. Hinweis des BfArM und des RKI zu komplex aufgebauten Endoskopen (Duodenoskopen), deren Aufbereitung und damit verbundenen Infektionsrisiken. German. Available from: https://www. bfarm.de/SharedDocs/Risikoinformationen/Medizinprodukte/DE/ bfarm_rki_aufbereitung_endoskop.html [Accessed: April 20 2017]

[11] Humphries RM, McDonnell G. Superbugs on duodenoscopes: the challenge of cleaning and disinfection of reusable devices. J Clin Microbiol 2015; 53: 3118-3125

[12] Petersen BT. Duodenoscope reprocessing: risk and options coming into view. Gastrointest Endosc 2015; 82: 484-486

[13] Gastmeier P, Vonberg RP. Klebsiella spp. in endoscopy-associated infections: we may only be seeing the tip of the iceberg. Infection 2014; 42: $15-21$

[14] Kim S, Russell D, Mohamadnejad M et al. Risk factors associated with the transmission of carbapenem-resistant Enterobacteriaceae via contaminated duodenoscopes. Gastrointest Endosc 2016; 83: 1121 1129

[15] Guyatt GH, Schünemann HJ, Djulbegovic B et al. Guideline panels should not GRADE good practice statements. J Clin Epidemiol 2015; 68: $597-600$

[16] Guyatt GH, Alonso-Coello P, Schünemann HJ. Guideline panels should seldom make good practice statements: guidance from the GRADE Working Group. J Clin Epidemiol 2016; 80: 3-7

[17] Dumonceau JM, Hassan C, Riphaus A et al. European Society of Gastrointestinal Endoscopy (ESGE) Guideline Development Policy. Endoscopy 2012; 44: 626-629

[18] Rutala WA, Weber DJ. Gastrointestinal endoscopes: a need to shift from disinfection to sterilization? JAMA 2014; 312: 1405-1406 [doi:10.1001/jama.2014.12559]

[19] Jung M, Beilenhoff U. Hygiene: The looming Achilles heel in endoscopy. Visc Med 2016; 32: 21-28

[20] Ross AS, Baliga C, Verma P et al. A quarantine process for the resolution of duodenoscope-associated transmission of multidrug-resistant Escherichia coli. Gastrointest Endosc 2015; 82: 477-483

[21] Beilenhoff U, Neumann CS, Rey JF et al. ESGE-ESGENA guideline: cleaning and disinfection in gastrointestinal endoscopy. Endoscopy 2008; 40: 939-957

[22] Association for the Advancement of Medical Instrumentation. ANSI/ AAMI ST91: 2015. Flexible and semi-rigid endoscope processing in health care facilities. Available from: http://nascecme.com.br/2014/ wp-content/uploads/2015/09/ANSI-AAMI-ST91-Endoscopy-2015.pdf [Accessed: April 20 2017]

[23] American Society for Gastrointestinal Endoscopy. Multisociety guideline on reprocessing flexible $\mathrm{Gl}$ endoscopes: 2016 update. Gastrointest Endosc 2017; 85: 282e-294e
[24] Association of periOperative Registered Nurses (AORN). Guideline for processing flexible endoscopes. Guidelines for Perioperative Practice 2017: doi:10.6015/psrp.17.01.717

[25] Gastroenterological Society of Australia/Gastroenterological Nurses College of Australia (GESA/GENCA). Draft document for discussion. Infection control in endoscopy - Multidrug resistant outbreaks response. Version: April 2016. Available from: http://www.genca.org/ public/5/files/Infection\%20Control\%20Guidelines\%20Draft\%20April\% 202016.pdf [Accessed: April 20 2017]

[26] ESGENA. European Curriculum for reprocessing flexible endoscopes and endoscopic accessories 2017. Forthcoming 3 Nov 2017, at http://www.esgena.org/statements-curricula.html

[27] Council Directive 93/42/EEC of 14 June 1993 concerning medical devices (OJ L 169, 12.7.1993, p. 1). Available from: http://eur-lex.europa.eu/LexUriServ/LexUriServ.do?uri=CONSLEG:1993L0042:20071011:en:PDF [Accessed: September 22 2017]

[28] Roberts CG. The role of biofilms in reprocessing medical devices. Am ] Infect Control 2013; 41: S77 - S80

[29] Otter JA, Vickery K, Walker JT et al. Surface-attached cells, biofilms and biocide susceptibility: implications for hospital cleaning and disinfection. J Hosp Infect 2015; 89: 16 - 27

[30] Bertschinger P, Brenneisen M, Cathomas A et al. Schweizerische Richtlinie zur Aufbereitung flexibler Endoskope. Gemeinsame Richtlinie der Schweizerischen Gesellschaft für Gastroenterologie (SGG), Schweizerischen Gesellschaft für Pneumologie (SGP), Schweizerischen Gesellschaft für Spitalhygiene (SGSH), Schweizerischen Vereinigung für Endoskopiepersonal (SVEP). German. Available from: https://svep-aspe.ch/fileadmin/user_upload/pdf/Schweizerische_Hygienerichtlinie2013.pdf

[31] Ministère de la santé, de la famille et des personnes handicapées, DGS/DHOS, Comité Technique national des Infections Nosocomiales (CTIN). Bonnes pratiques de désinfection des dispositifs médicaux. Guide pour l'utilisation des laveurs-désinfecteurs d'endoscopes. French. November 2003. Available from: http://nosobase.chu-lyon. $\mathrm{fr} /$ recommandations/Ministere_Sante/2003_endoscopie_ministere. pdf [Accessed: September 22 2017]

[32] Sethi S, Huang RJ, Barakat M et al. Adenosine triphosphate bioluminescence for bacteriologic surveillance and reprocessing strategies for minimizing risk of infection transmission by duodenoscopes. Gastrointest Endosc 2017; 85: 1180-1187

[33] Ramsey AH, Oemig TV, Davis JP et al. An outbreak of bronchoscopyrelated Mycobacterium tuberculosis infections due to lack of bronchoscope leak testing. Chest 2002; 121: 976 - 981

[34] Seki M, Hashiguchi K, Tanaka A et al. Characteristics and disease severity of healthcare-associated pneumonia among patients in a hospital in Kitakyushu, Japan. Infect Chemother 2011; 17: 363-369

[35] Beilenhoff U, Neumann CS, Biering $\mathrm{H}$ et al. ESGE Guidelines Committee. ESGE/ESGENA guideline for process validation and routine testing for reprocessing endoscopes in washer-disinfectors, according to the European Standard prEN ISO 15883 parts 1, 4 and 5. Endoscopy 2007; 39: $85-94$

[36] Beilenhoff U, Biering H, Blum R et al. European Society of Gastrointestinal Endoscopy (ESGE)-European Society of Gastroenterology and Endoscopy Nurses and Associates (ESGENA). Technical Specification for process validation and routine testing of endoscope reprocessing in washer-disinfectors, according to EN ISO 15883, parts 1, 4, and ISO/TS 15883-5. Forthcoming Nov/Dec 2017

[37] Beilenhoff U, Neumann CS, Rey JF et al. ESGE-ESGENA guideline for quality assurance in reprocessing: microbiological surveillance testing in endoscopy. Endoscopy 2007; 39: 175-181

[38] Centers for Disease Control and Prevention. Interim duodenoscope surveillance protocol, updated April 3, 2015. Available from: http:// www.cdc.gov/hai/organisms/cre/cre-duodenoscope-surveillanceprotocol.html [Accessed: April 20 2017] 